\title{
Probiotic Efficacy of Microencapsulated Saccharomyces cerevisiae on Gastrointestinal Tract Integrity in Rats
}

\author{
Ali Reza Khosravi ${ }^{1,2} \mathbb{D}$, Hassan Ghorbani-Choboghlo ${ }^{1,2}$, Donya Nikaein ${ }^{1,2}$ (D) , Hadi Pourjafar ${ }^{3,4 *(\mathbb{D})}$ \\ 1 Mycology Research Center, Faculty of Veterinary Medicine, University of Tehran, Tehran, Iran, khosravialir@gmail.com \\ (A.R.K.); donya.nik@gmail.com (D.N.); hghorbani@ut.ac.ir (H.G.C.); \\ 2 Department of Microbiology and Immunology, Faculty of Veterinary Medicine, University of Tehran, Tehran, Iran \\ 3 Department of Food Sciences and Nutrition, Maragheh University of Medical Sciences, Maragheh, Iran, \\ pourjafarhadi59@gmail.com (H.P.); \\ 4 Alborz University of Medical Sciences, Dietary Supplements and Probiotic Research Center, Karaj, Iran \\ * Correspondence: pourjafarhadi59@gmail.com;
}

Received: 9.08.2020; Revised: 8.09.2020; Accepted: 10.09.2020; Published: 13.09.2020

\begin{abstract}
Saccharomyces cerevisiae as a probiotic has been prescribed for prophylaxis and treatment of gut infected diseases. This study was designed to assess the effects of encapsulated S. cerevisiae on gastrointestinal tract properties in the animal model. In rats, after 8 -week feeding by encapsulated and unencapsulated $S$. cerevisiae, the mount of the IgA protein was determined by ELISA. Rats were euthanized, and the liver, kidney, and intestinal tract were collected for histological analysis. The consumption of S. cerevisiae could increase IgA levels in comparison with the control group. This increase was significant in the lower parts of the small intestine $(\mathrm{p}<0.05)$. In histopathological evaluations; Liver microscopic examination showed fatty change and margination of Kupffer cells as well as their hyperplasia and hypertrophy, which is a mark for liver regeneration in both groups that received microencapsulated and free probiotic. In spleen structure, in both groups, mild inflammation of the spleen tissue in the form of accumulation of red pulp of erythrocytes, hypercellular of this tissue was observed due to hyperplasia of lymphoid follicles and hyperplasia and hepaticophyta of retinal cells and macrophages. The lymphatic structure of the spleen showed relatively intense hyperplasia. In the colon structure, in both groups, hyperplasia of goblet calls along with slight infiltration of inflammatory cells was noted. Calcium alginate encapsulation considerably improves the yeast viability in simulated gastric juice and simulated intestine juice situations. Also, S. cerevisiae has positive profits in suitable food absorption and then decreasing diarrhea and other similar gastrointestinal disorders.
\end{abstract}

Keywords: Probiotic; Saccharomyces cerevisiae; Encapsulation; gastrointestinal condition; Ig A; Histopathological.

(C) 2020 by the authors. This article is an open-access article distributed under the terms and conditions of the Creative Commons Attribution (CC BY) license (https://creativecommons.org/licenses/by/4.0/).

\section{Introduction}

Probiotics are live microorganisms that, when administered in an adequate amount, confer one or more specific confirmed health profits on the consumer through the gut tract [1]. Saccharomyces cerevisiae is an accurate probiotic yeast, and some of the nonpathogenic serotypes of this microorganism has been prescribed for prophylaxis and treatment of gut infected diseases during the past decade [1]. The effects of $S$. cerevisiae on intestinal microflora and enteric pathogens (or their toxins), including Escherichia coli, has been reported in various studies [2,3]. Actually, controlled clinical trials have indicated that oral consumption of $S$. cerevisiae var. boulardii (S. boulardii) could cure or inhibit gastrointestinal (GI) disease, for 
instance, periodic Clostridium difficile-associated syndrome, children acute diarrhea, antibiotic-associated diarrhea, Traveler's diarrhea, AIDS-associated diarrhea, irritable bowel syndrome, and Crohn's disease. Some studies have shown that there is a close interaction between luminal flora and the apical aspect of ileal surface enterocytes in rats [4,5]. Immune cells in the GI lumen should respond quickly to invasive pathogens but do not hurt commensal microflora as well. Indeed, salivary $\operatorname{IgA}(\mathrm{SIgA})$ is regarded as a health biomarker to evaluate the risk of gut infections associated with acute and chronic gut diseases [6].

Generally, Till now, Saccharomyces spp. has been found to act in two principle ways; first, it inhibits some bacterial toxins or their effects, and secondly, it has direct effects on the intestinal mucosa [7,8]. However, there are still difficulties encountered with the use of probiotics in foods; one of them is the viability rate of the probiotics in foods and during GI passage to the location of action in the human gut.

Different agents like exposure to acid, bile, and osmotic and oxidative stresses may decrease the number of probiotics under the effective threshold. Then, different techniques are available for improving the survival of probiotic microorganisms, and microencapsulation is one of the best and most outstanding methods, and this technique can be effective in both product storage as well as GI condition $[9,10]$.

Microencapsulation via calcium alginate (an anionic linear heteropolysaccharide) is an effectual method for the immobilization of probiotic microorganisms. The easiness of usage, its non-toxic nature, and its low price have made it one of the most broadly used materials for microencapsulation $[11,12]$. This study was designed to evaluate the effects of encapsulated $S$. cerevisiae on GI lumen properties in the animal model (in rats).

\section{Materials and Methods}

\subsection{Yeast strain and growth conditions.}

The yeast strain employed in this investigation was $S$. cerevisiae (ATCC code 9763). Yeasts were activated by culturing cells from refrigerated slant on $50 \mathrm{ml}$ Subouraud Dextrose (SD) broth medium (Merck Co., Darmstadt, Germany) and incubating overnight at $28{ }^{\circ} \mathrm{C}$ with orbital shaking of $180 \mathrm{rpm}$. After that, $1 \mathrm{ml}$ of the cultured suspension was inoculated into another $50 \mathrm{ml}$ flask containing the same medium and incubated for $24 \mathrm{~h}$ under the same culture conditions. Consequently, the cells of $S$. cerevisiae were harvested by centrifugation at 10,000 rpm for $10 \mathrm{~min}$ and washed two times with sterile phosphate-buffered saline (PBS) to remove the culture medium from the cells thoroughly. Finally, harvested yeast cells were counted and used in the microencapsulation process.

\subsection{Microencapsulation procedure.}

In this investigation, the extrusion technique with sodium alginate and calcium chloride was carried out for encapsulation. In brief, a $4 \% \mathrm{Na}$-alginate mixture in distilled water was made, and then, the mixture of cell suspension and $\mathrm{Na}$-alginate were injected into a $0.1 \mathrm{M}$ $\mathrm{CaCl} 2$ solution through a sterile insulin syringe. In the end, the droplets formed gel spheres immediately, and the produced beads were gathered and rinsed with distilled water and used on the same day [13-15]. 


\subsection{Preparation of simulated gastric and intestinal juices.}

Simulated gastric juices (SGJ) were prepared by suspending pepsin (P7000, 1:10,000) in sterile sodium chloride solution $(0.5 \%, \mathrm{w} / \mathrm{v})$ to a final concentration of $3 \mathrm{~g} \mathrm{~L}-1$ (1038 U mL1) and adjusting the $\mathrm{pH}$ to 2.0 with concentrated $\mathrm{HCl}$ or sterile $0.1 \mathrm{~mol} \mathrm{~L}-1 \mathrm{NaOH}$. Simulated intestinal juices (SIJ) were prepared by suspending pancreatin USP (P-1500) in sterile sodium chloride solution $(0.5 \%, \mathrm{w} / \mathrm{v})$ to a final concentration of $1 \mathrm{~g} \mathrm{~L}-1$, with $4.5 \%$ bile salts (Oxoid, Basingstoke, UK) and adjusting the $\mathrm{pH}$ to 8.0 with sterile $0.1 \mathrm{~mol} \mathrm{L-1} \mathrm{NaOH}$. Both solutions were filtered for sterilization through a $0.22 \mu \mathrm{m}$ membrane $[16,17]$.

\subsection{Cell tolerance to the simulated gastrointestinal condition and determination of total viable} counts.

The tolerance of free and encapsulated cells of $S$. cerevisiae on SGJ and SIJ was determined using the modified method. The tests were performed using a series of $15 \mathrm{~mL}$ sterile falcon tubes. Two different conditions were tested: in the first and the second examinations, $0.4 \mathrm{~mL}$ of the suspension of either encapsulated or free yeasts were mixed with $1.8 \mathrm{~mL}$ of SGJ or SIJ, lightly blended, and incubated at $28{ }^{\circ} \mathrm{C}$ for $120 \mathrm{~min}$. The control for these tests was done by incubating $0.4 \mathrm{~mL}$ of either free or encapsulated yeasts in $1.8 \mathrm{~mL}$ sterile sodium chloride solution $(0.5 \%, \mathrm{w} / \mathrm{v})$ at $28{ }^{\circ} \mathrm{C}$ for $120 \mathrm{~min}$. After the addition of free or encapsulated yeasts to SGJ and SIJ, the pH range of these was corrected to 2.0 and 8.0, respectively, with sterile 0.1 mol L-1 Sodium hydroxide or concentrated hydrochloric acid. Then, aliquots of $1 \mathrm{~mL}$ were removed at $0,30,60$, and 120 min to determine the total viable counts of $S$. cerevisiae via the pour plate method using SD agar (Merck Co., Darmstadt, Germany) and serial 10-fold dilutions in peptone water (PW). Plates were incubated at $28^{\circ} \mathrm{C}$ for $72 \mathrm{~h}$ and in a dark cabinet [16-18].

\subsection{Animals and in vivo procedures.}

The study protocol was approved by the Research Ethics Committee of Tehran University. All animal experiments were carried out in accordance with the U.K. Animals (Scientific Procedures) Act, 1986 and associated guidelines.

Thirty-day old male Wistar rats, weighing about $70 \mathrm{~g}$, were used in this study. During this period, the intestinal mucosa was still immature, and the production of the secretory component of immunoglobulin (SC) was very low compared to adult rats. Likewise, it was compulsory to avoid the suckling period for the reason that rat milk is an exogenous source of s-IgA.

All animals were kept in polystyrene cages in an air-conditioned room at $21 \pm 1^{\circ} \mathrm{C}$ and $12 \mathrm{~h}$ light-dark cycles. Standard pellet food and tap water were provided for all rats. Animals were adapted to experimental situations for one week prior to examination. Rats were divided into three groups, each containing 6 male rats: rats receiving encapsulated $S$. cerevisiae, rats receiving unencapsulated $S$. cerevisiae, and control group. $S$. cerevisiae as a probiotic was gavaged to rats at the dosage of $2 \mathrm{~g} / \mathrm{kg}$ for 8 weeks, and the control group was treated with normal saline.

Finally, rats were weighed individually, and feed consumption was measured weekly throughout the 8-week experiment. There were three groups of rats for tests as follows:

Group a) control, by normal diet and gavage with distilled water (6 male rats).

Group b) by normal diet and daily gavage with free probiotic emulsion $(1 \times 108 \mathrm{cfu} \mathrm{ml}-1)(6$ male rats). 
Group c) by normal diet and daily gavage with encapsulated probiotic emulsion $(1 \times 108 \mathrm{cfu}$ ml-1) (6 male rats).

\subsection{Histology of intestinal tract, kidneys, and liver.}

Rats were euthanized at the end of the 8th week. The liver, kidney, and intestinal tract were collected for histological analysis. Intestinal samples included segments of approximately $2 \mathrm{~cm}$ from the mid-point of the duodenum, $2 \mathrm{~cm}$ from the mid-point of ileum, and $10 \mathrm{~cm}$ proximal to the ileocecal junction. Samples were fixed in $10 \%$ formalin solution and embedded in paraffin wax. All histological investigations were carried out on $5 \mathrm{ml}$ segments, stained by hematoxylin and eosin ( $\mathrm{H} \& \mathrm{E})$, and observed by means of an Olympus AX70 microscope (Olympus Cooperation, Tokyo, Japan) equipped with a digital video camera (Sony DXC930P). In the duodenum and ileum (four cross-sections for each sample), the villus length was measured from the villus tip to the villus-crypt junction, while crypt depth was defined as the depth of the invagination between two villi. Sections $4 \mathrm{~m}$ thick were stained with hematoxylin and eosin and analyzed by a pathologist who was blinded to the groups.

\subsection{Detection of the SIgA protein by ELISA.}

Intestinal mucosa was isolated, according to Jarillo-Luna et al. (2007) method with brief modifications. In brief, intestinal content was washed out with cold PBS containing penicillin/streptomycin $(100 \mu \mathrm{g} / \mathrm{ml})$ and centrifuged at $500 \mathrm{~g}$ for $10 \mathrm{~min}$. IgA was measured in duodenal and jejunal washings was measured using ELISA assay according to the manufacturer's instructions [19].

\subsection{Statistical analysis.}

Data are presented as the mean $\pm \mathrm{SD}$ of three independent assays (6 animals per group, per assay). The levels of valuable probiotic were analyzed by one-way ANOVA, followed by a Holm-Sidak method post hoc test. Immunological data were analyzed with two-way ANOVA for the examined factor (between groups) and intestinal region (within each group). If a significant main effect or association was identified $(\mathrm{p}<0.05)$, the respective group means were compared using the Holm-Sidak method. For all tests, $\mathrm{p}<0.05$ was considered significant. All analyses were performed using the statistical program Sigma Stat for Windows Version 2.03 software (SPSS Inc.).

\section{Results and Discussion}

\subsection{Size and shape of beads.}

Surface morphology, size, and figure of the 50 randomly selected Ca-alginate beads were determined by means of light microscopy at a magnification of $40 x$. The figure of the beads was commonly spherical, sometimes elliptical, with a mean diameter of 100-200 $\mu \mathrm{m}$ (See Fig. 1). The loss throughout encapsulation was very low because of the gentle techniques employed. 


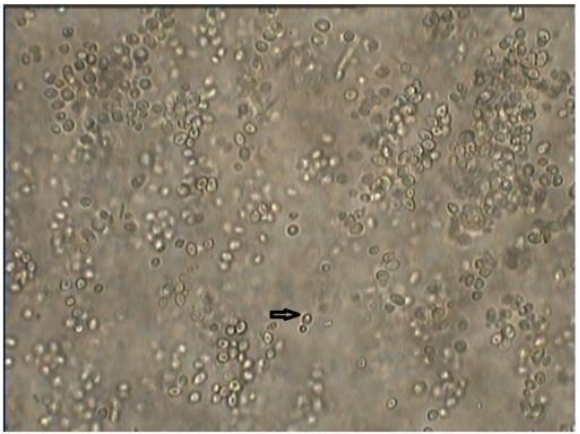

Figure 1. The shape of the calcium alginate beads was generally spherical, with a mean diameter of 50-150 $\mu \mathrm{m}$.

\subsection{Cell tolerance to the simulated gastrointestinal conditions.}

The survival rate of encapsulated yeast cells in SGJ, and SIJ solutions are demonstrated in Table 1. No significant decrease in the viable count was detected in free as well as encapsulated yeasts in distilled water $(\mathrm{pH}$ 6.5) after incubation for $2 \mathrm{~h}$ (control) ( $\mathrm{p}>0.05)$. Nonetheless, variations in numbers of free and encapsulated $S$. cerevisiae throughout incubation in SGJ and SIJ were significant (Table 1). Even though consequences revealed that there were considerable declines in free yeasts in immediate exposure to $\mathrm{pH} 2$ and 8 , after 120 min incubation in SGJ and SIJ circumstances. There was a significant rise in the survival rate of yeast cells in the capsules compared to free cells $(\mathrm{p}<0.05)$.

Table 1. Number of viable cells after exposure to SGI and SIJ for different times (Mean \pm SD).

\begin{tabular}{c|c|c|c|c|c|c}
\multirow{2}{*}{ Condition } & \multirow{2}{*}{ Type of cell } & \multicolumn{5}{|c}{ Hardening time (min) } \\
\cline { 3 - 7 } & & $\mathbf{0}$ & $\mathbf{3 0}$ & $\mathbf{6 0}$ & $\mathbf{9 0}$ & $\mathbf{1 2 0}$ \\
\hline \multirow{2}{*}{ distilled water } & free & $9 \pm 0.2 \times 10^{10}$ & $9 \pm 0.4 \times 10^{10}$ & $9 \pm 0.4 \times 10^{10}$ & $8 \pm 0.1 \times 10^{10}$ & $8 \pm 0.3 \times 10^{10}$ \\
\cline { 2 - 7 } & Capsulated & $9 \pm 0.2 \times 10^{10}$ & $9 \pm 0.3 \times 10^{10}$ & $9 \pm 0.2 \times 10^{10}$ & $8 \pm 0.4 \times 10^{10}$ & $7 \pm 0.4 \times 10^{10}$ \\
\hline \multirow{2}{*}{ SGJ } & free & $7 \pm 0.2 \times 10^{10}$ & $4 \pm 0.1 \times 10^{9}$ & $6 \pm 0.3 \times 10^{8}$ & $7 \pm 0.1 \pm \times 10^{7}$ & $2 \pm 0.2 \times 10^{6}$ \\
\cline { 2 - 7 } & Capsulated & $8 \pm 0.3 \times 10^{10}$ & $7 \pm 0.2 \times 10^{10}$ & $2 \pm 0.3 \times 10^{10}$ & $5 \pm 0.7 \times 10^{9}$ & $2 \pm 0.6 \times 10^{8}$ \\
\hline \multirow{2}{*}{ SIJ } & free & $7 \pm 0.3 \times 10^{10}$ & $5 \pm 0.2 \times 10^{9}$ & $7 \pm 0.4 \times 10^{8}$ & $3 \pm 0.3 \times 10^{8}$ & $5 \pm 0.2 \times 10^{6}$ \\
\cline { 2 - 7 } & Capsulated & $8 \pm 0.4 \times 10^{10}$ & $7 \pm 0.2 \times 10^{10}$ & $3 \pm 0.2 \times 10^{10}$ & $8 \pm 0.1 \times 10^{9}$ & $5 \pm 0.1 \times 10^{8}$
\end{tabular}

\subsection{Effect of microencapsulated probiotic on the weight of different organs.}

The rats in the encapsulated group (group c) mice had gained more weight. The studied organ weight was higher in the treatment group as well; nevertheless, weight gain was not significant between groups $(\mathrm{p}>0.05)$. (See Table 2$)$

Table 2. Effects of probiotic consumption on the weight of mice and some internal organs (Mean \pm SD).

\begin{tabular}{c|c|c|c|c|c} 
& Weight $\mathbf{( g )}$ & & & & \\
\hline & Total weight & Liver & Spleen & Lung and heart & Stomach and intestine \\
\hline Group a & $154.6 \pm 0.1$ & $2.98 \pm 0.3$ & $0.33 \pm 0.1$ & $1.6 \pm 0.2$ & $14.6 \pm 0.4$ \\
\hline Group b & $169.6 \pm 0.2$ & $4.16 \pm 0.5$ & $0.29 \pm 0.4$ & $1.88 \pm 0.7$ & $16.22 \pm 0.5$ \\
\hline Group c & $180.4 \pm 0.1$ & $4.66 \pm 0.4$ & $0.41 \pm 0.1$ & $2.26 \pm 0.1$ & $17.4 \pm 0.3$
\end{tabular}

\subsection{Secretory IgA measurement.}

Assessment of IgA levels in 1/8 of beginning (Duodenum) and lower parts (Jejunum) of the small intestine disclosed that the eating of probiotic (S. cerevisiae) in both groups $\mathrm{b}$ and c could rise secretory IgA levels compared to the group a (or control). This increase was significant in the lower parts of the small intestine $(\mathrm{p}<0.05)$ (See Table 3$)$. 
Table 3. Effects of probiotic consumption on secretory IgA changes in duodenum and jejunum.

\begin{tabular}{c|c|c} 
& IgA $(\mathbf{n g} / \mathbf{m l})$ & \\
\hline & Duodenum & Jejunum \\
\hline Group a & 288 & 192 \\
\hline Group b & 488 & 482 \\
\hline Group c & 610 & 541.5
\end{tabular}

\subsection{Histopathological findings.}

No significant changes were observed in the gross examination of GI lumen, urinary, respiratory, and CNS systems, and microscopic assessment of kidneys only illustrated insignificant hyperemia in medulla and cortex sections. Liver microscopic examination displayed fatty change and margination of Kupffer cells as well as their hyperplasia and hypertrophy, which is a mark for liver regeneration in the group b and $\mathrm{c}$ of rats (that received encapsulated and free probiotics) (See Fig. 2a and Fig. 2b).

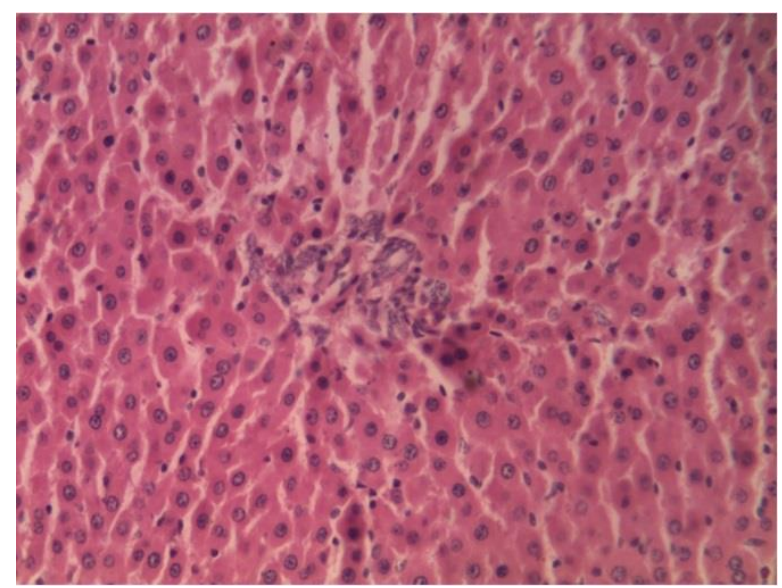

Figure 2a. Normal liver structure in control rats (H\&E 100x).

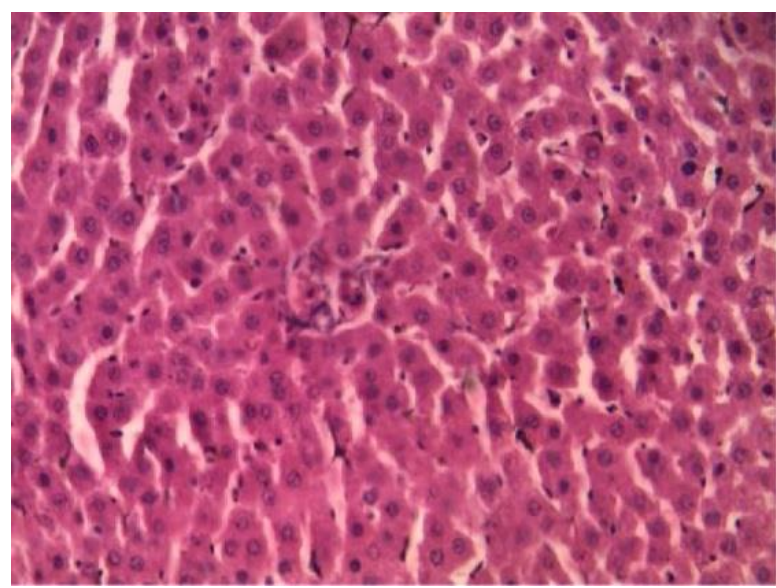

Figure 2b. Liver structure in the group b and c (that received microencapsulated and free probiotics) was similar, and in both groups, the severity of the reactivation was higher compared to the control group (H\&E $100 \times)$.

In spleen structure, in the group b and c, mild inflammation of the spleen tissue in the form of accumulation of red pulp of erythrocytes, hypercellular of this tissue was observed due to hyperplasia of lymphoid follicles and hyperplasia and hepaticophyta of retinal cells and macrophages. The lymphatic structure of the spleen showed relatively intense hyperplasia (fully expanded with PALS density) (See Fig. 3a and Fig. 3b). 


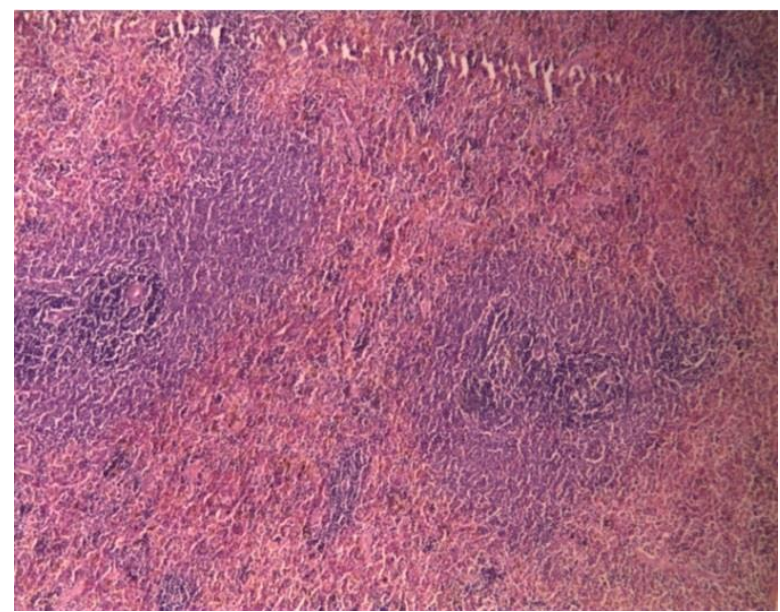

Figure 3a. Normal spleen structure in control rats (H\&E 100x).

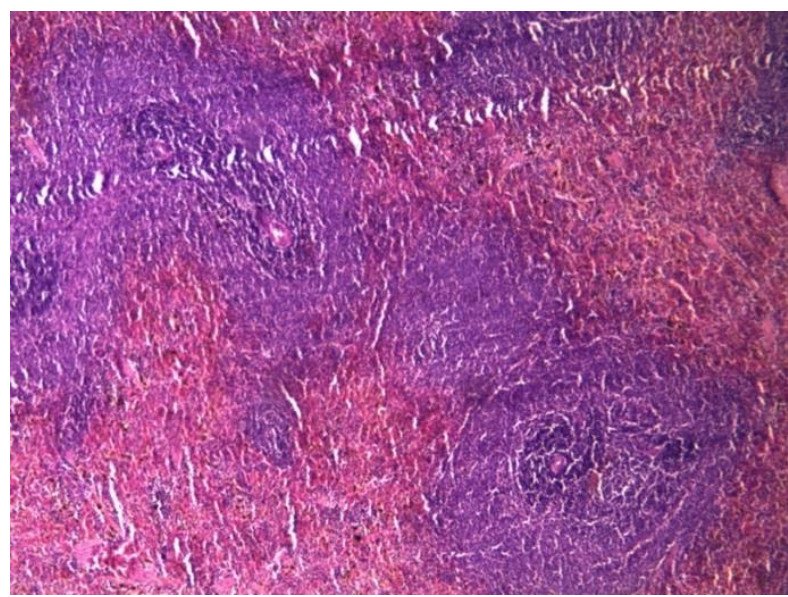

Figure 3b. Spleen structure in the group b and c (that received microencapsulated and free probiotics) was similar, and in both groups, mild spleen hyperemia and relatively severe spleen lymphatic hyperplasia was seen (H\&E 100x).

In the colon structure, in the group b and $\mathrm{c}$ of rats (that received encapsulated and free probiotics), hyperplasia of goblet calls along with slight infiltration of inflammatory cells was noted (see Fig. 4a and Fig. 4b).

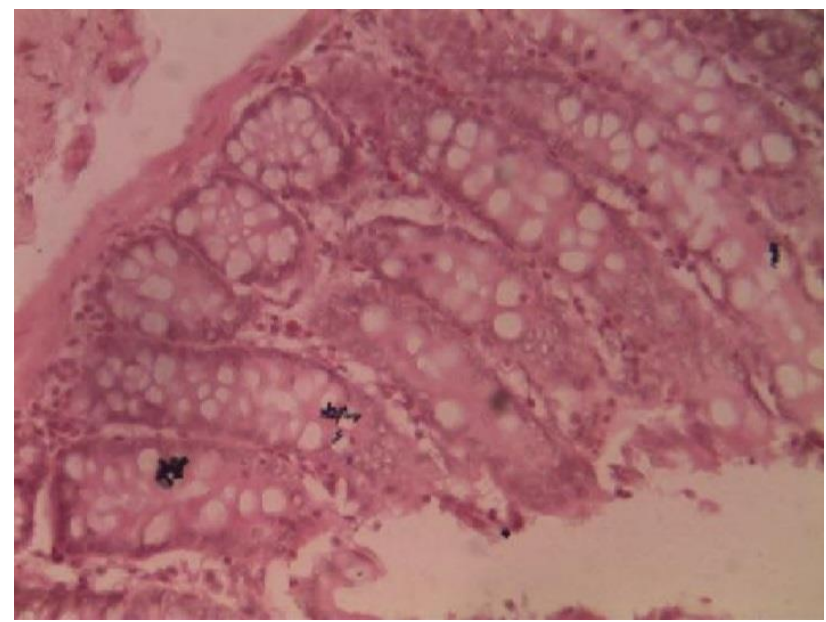

Figure 4a. Normal colon structure in control rats (H\&E 100x). 


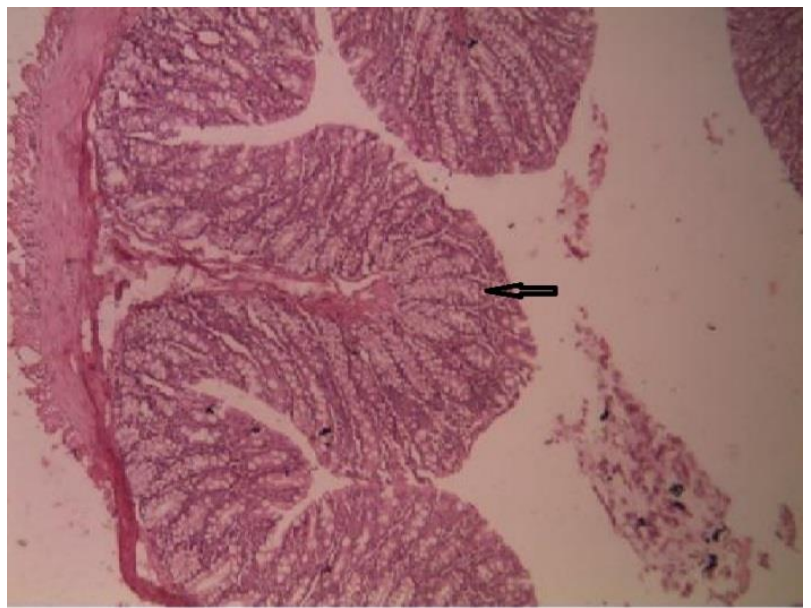

Figure 4b. Colon structure in the group b and c (that received microencapsulated and free probiotics) was similar, and in both groups, Goblet cells hyperplasia and increased height of the intestinal folds were seen (H\&E $100 \times)$.

Over the years, probiotic microorganisms are achieving scientific and commercial interest and are currently quite ordinary in our daily life, starting from health-promoting useful foodstuffs to therapeutic, prophylactic, and growth supplements [20,21]. Saccharomyces is extensively employed for the inhibition of diarrhea and other GI disturbances caused by the consumption of different antibiotics. This yeast can stay viable all along in the GI lumen. On the contrary to prokaryotic cells, the utilization of eukaryotic as probiotics is limited, and merely a few probiotics of eukaryotic origin are commercially accessible for human and animal practices.

Viability of the S. cerevisiae was found to rise on alginate encapsulation. Furthermore, there was no significant difference in the survivability of free yeasts in distilled water, showing that water had no influence on survival $[22,23]$.

Among the eukaryotic probiotics, yeasts, particularly Saccharomyces species, are leading and regularly employed in a wide range of hosts that can endure a wide range of temperature, salt concentration, and $\mathrm{pH}$ depending upon the strain, mainly S. cerevisiae [24]. Moreover, in this investigation, the number of viable probiotic yeasts achieved for all the capsules was above the recommended levels for a probiotic foodstuff, i.e., equal to or greater than 6-log CFU g-1 of the product, which is in accordance with former reports [24,25].

On the other hand, the free strain showed a steady loss in viability when exposed to acid conditions. However, the microcapsules containing $S$. cerevisiae survived very well $(\mathrm{P}<$ $0.05)$ after exposure to in vitro acid conditions when compared with the free cells. Furthermore, a decline of roughly 4-log was noted in the number of free cells after $2 \mathrm{~h}$ of incubation at $\mathrm{pH}$ 2 , once compared to declines of approximately 2-log in the all encapsulated $S$. cerevisiae beneath similar situations $[20,26]$.

Nonetheless, the articles report mentioned the effect of OX gall bile on the survivability of the probiotic microorganisms; in this study, the capsulate yeast has a significant survival rate in SIJ. Lately, Saccharomyces probiotics have been found to aid in controlling the pathogenesis of diabetes and other metabolic illnesses. It is compulsory to declare that hightolerance of yeast to diverse situations, for instance, gastric, intestinal, and into food throughout storage, displayed the worthy potential of this microorganism as a probiotic. Regarding the about mentioned points and agreement of this study consequences with several other reports about the encapsulation of bacteria, $\mathrm{Ca}$ alginate could be good material for the protection of probiotic cells [27,28]. 
The weighted parameters showed that the groups receiving the probiotics (especially in the group c) had significantly higher weight gain over the two months (Weight gain in the intestines can be indicative of a significant increase in the intestinal villi and cells). This matter could be a reason for the positive benefits of probiotics in suitable food absorption and then reducing diarrhea and other similar GI disorders.

Certainly, the most important issue in the efficiency and health of the GI lumen is the existence of a healthy immune system and mucosal integrity. The secretion of mucosal immunoglobulin and cytokines is one of the main strategies to prevent the attachment and penetration of pathogenic microbes. The present study showed that feeding the mice with probiotics, and especially the microencapsulated type, can significantly increase the amount of mucosal IgA secretion. In a similar study by Buts and Keyser (2010), mucosal immunoglobulin levels were increased by about $150 \mathrm{ng} / \mathrm{mL}$ after administration of Saccharomyces yeast to mice. However, in the present study, it seems that due to the microencapsulation of Saccharomyces and its high-level presence in the intestine, mucosal immunoglobin secretion was increased dramatically [29].

Results of some studies have shown that constant use of probiotic strains can enhance the ability of the GI lumen in rats. Conrads et al. (2018) showed that 12 weeks of consumption of probiotics could improve the negative changes that occur after various GI lumen injuries. They indicated that in addition to strengthening the immune system of the GI lumen, the process of regenerating intestinal villi also increased [30]. In the present study, a 2-week diet of rats fed probiotics showed that in addition to enhancing the mucosal immune cells, the rate of growth and remodeling of the intestinal villi increased broadly. The rate of these changes was similar in the b and c groups, but both groups were significantly different from the control group.

\section{Conclusions}

S. cerevisiae yeast cells, important in the fermentation industry, involve a natural, foodgrade, inexpensive, and abundant food material. The present study has demonstrated that calcium alginate encapsulation considerably improves the yeast viability in SGJ and SIJ situations and permit viable cells to reach a useful level as probiotic. In order to get the other aspect of yeast probiotics, this study should be continued by different materials and in functional food conditions with the defined variable into the further.

\section{Funding}

This research received no external funding.

\section{Acknowledgments}

This work was supported by the Research Council of the University of Tehran. The results described in this paper were part of a student thesis. The authors would like to thank Mr Asad Balal for his kind cooperation during the project.

\section{Conflicts of Interest}

The authors declare that there is no conflict of interest. 


\section{Human and Animal Rights}

The study protocol was approved by the Research Ethics Committee of Tehran University. All animal experiments were carried out in accordance with the U.K. Animals (Scientific Procedures) Act, 1986 and associated guidelines.

\section{References}

1. Moayyedi, P.; Ford, A.C.; Talley, N.J.; Cremonini, F.; Foxx-Orenstein, A.E.; Brandt, L.J.; Quigley, E.M. The efficacy of probiotics in the treatment of irritable bowel syndrome: a systematic review. Gut 2010, 59, 325-332, https://doi.org/10.1136/gut.2008.167270.

2. Lima Filho, J.; Vieira, E.; Nicoli, J. Antagonistic effect of Lactobacillus acidophilus, Saccharomyces boulardii and Escherichia coli combinations against experimental infections with Shigella flexneri and Salmonella enteritidis subsp. typhimurium in gnotobiotic mice. J Appl Microbiol 2000, 88, 365-370, https://doi.org/10.1046/j.1365-2672.2000.00973.x.

3. Behcet, M.; Kaya, A.D. Investigation of therapeutic effect of Saccharomyces boulardii and translocation in immunsupressed rats infected with Shigella sonnei. Expert Biomed Res 2020, 3, 141-148, https://doi.org/10.30714/j-ebr.2020361049.

4. Sorribas, M.; Jakob, M.O.; Yilmaz, B.; Li, H.; Stutz, D.; Noser, Y.; de Gottardi, A, Moghadamrad, S.; Hassan, M.; Albillos, A.; Francés, R. Intestinal mucus and gut-vascular barrier: FxR-modulated entry sites for pathological bacterial translocation in liver cirrhosis. BioRxiv 20191.

5. Lucius, K. Dietary Lectins: Gastrointestinal and Immune Effects. Altern Complement Ther 2020, 26, 16874, https://doi.org/10.1089/act.2020.29286.klu.

6. Agatonovic-Kustrin, S.; Morton, D.W.; Smirnov, V.; Petukhov, A.; Gegechkori, V.; Kuzina, V.; Gorpinchenko, N.; Ramenskaya, G. Analytical strategies in lipidomics for discovery of functional biomarkers from human saliva. Dis markers 2019, 2019, https://doi.org/10.1155/2019/6741518.

7. Czerucka, D.; Rampal, P. Diversity of Saccharomyces boulardii CNCM I-745 mechanisms of action against intestinal infections. World J Gastroenterol 2019, 25, 2188-2203.

8. Plaza-Diaz, J.; Ruiz-Ojeda, F.J.; Gil-Campos, M.; Gil A. Mechanisms of action of probiotics. Adv Nutr 2019, 10, S49-66, https://doi.org/10.1093/advances/nmy063.

9. Bevilacqua, A.; Campaniello, D.; Speranza, B.; Racioppo, A.; Altieri, C.; Sinigaglia, M.; Corbo, M.R. Microencapsulation of Saccharomyces cerevisiae into Alginate Beads: A Focus on Functional Properties of Released Cells. Foods 2020 9, https://doi.org/10.3390/foods9081051.

10. Ghorbani-Choboghlo, H.; Nikaein, D.; Khosravi, A.R.; Rahmani, R.; Farahnejad, Z. Effect of microencapsulation on Saccharomyces cerevisiae var. boulardii viability in the gastrointestinal tract and level of some blood biochemical factors in wistar rats. Iran J Microbiol 2019, 11, 160-165.

11. Cao, L.; Xu, Q.; Xing, Y.; Guo, X.; Li, W.; Cai, Y. Effect of skimmed milk powder concentrations on the biological characteristics of microencapsulated Saccharomyces cerevisiae by vacuum-spray-freeze-drying. Drying Technol 2020, 38, 476-494, https://doi.org/10.1080/07373937.2019.1581797.

12. Hou, D.; Yu, W.; Zhang, D.; Zhao, L.; Liu, X.; Ma, X. Culture of yeast cells immobilized by alginatechitosan microcapsules in aqueous-organic solvent biphasic system. J Oceanol Limnol 2019, 37, 863-870, https://doi.org/10.1007/s00343-019-8126-9.

13. Ghasemnezhad, R.; Razavilar, V.; Pourjafar, H.; Khosravi-Darani, K.; Ala, K. The viability of free and encapsulated Lactobacillus casei and Bifidobacterium animalis in chocolate milk, and evaluation of its $\mathrm{pH}$ changes and sensory properties during storage. Ann Res.Rev Biol 2017, 1-8.

14. Pourjafar, H.; Noori, N.; Gandomi, H.; Basti, A.A.; Ansari, F. Stability and efficiency of double-coated beads containing lactobacillus acidophilus obtained from the calcium alginate-chitosan and Eudragit S100 nanoparticles microencapsulation. Int J Probiotics Prebiotics 2018, 13, 77-84.

15. Abdolhosseinzadeh, E.; Dehnad, A.R.; Pourjafar, H.; Homayouni, A.; Ansari, F. The production of probiotic Scallion Yogurt: Viability of Lactobacillus acidophilus freely and microencapsulated in the product. Carpath J Food Sci Technol 2018, 10, 72-80.

16. Ansari, F.; Pourjafar, H.; Jodat, V.; Sahebi, J.; Ataei, A. Effect of Eudragit S100 nanoparticles and alginate chitosan encapsulation on the viability of Lactobacillus acidophilus and Lactobacillus rhamnosus. $A M B$ Express 2017, 7, https://doi.org/10.1186/s13568-017-0442-x.

17. Mirzaei, H.; Pourjafar, H.; Rad, A.H. The effect of microencapsulation with calcium alginate and resistant starch on the Lactobacillus acidophilus (La5) survival rate in simulated gastrointestinal juice conditions. $J$ Vet Res 2011, 66, 337-377.

18. Pourjafar, H.; Noori, N.; Gandomi, H.; Basti, A.A. Study of protective role of double coated beads of calcium alginate-chitosan-eudragit S100 nanoparticles achieved from microencapsulation of Lactobacillus acidophilus as a predominant flora of human and animals gut. $J$ Vet Res 2016, 71, 311-320. 
19. Jarillo-Luna, A.; Rivera-Aguilar, V.; Garfias, H.R.; Lara-Padilla, E.; Kormanovsky, A.; Campos-Rodríguez, R. Effect of repeated restraint stress on the levels of intestinal IgA in mice. Psychoneuroendocrinology 2007, 32, 681-692, https://doi.org/10.1016/j.psyneuen.2007.04.009.

20. Morales-Amparano, M.B.; Montfort, G.R.; Baqueiro-Peña, I.; del Refugio Robles-Burgueño, M.; VázquezMoreno, L.; Huerta-Ocampo, J.Á. Proteomic response of Saccharomyces boulardii to simulated gastrointestinal conditions and encapsulation. Food Sci Biotechnol 2019, 28, 831-840, https://doi.org/10.1007/s10068-018-0508-9.

21. Fietto, J.L.; Araújo, R.S.; Valadão, F.N.; Fietto, L.G.; Brandão, R.L.; Neves, M.J.; Gomes, F.C.; Nicoli, J.R.; Castro, I.M. Molecular and physiological comparisons between Saccharomyces cerevisiae and Saccharomyces boulardii. Can J Microbiol 2004, 50, 615-621, https://doi.org/10.1139/w04-050.

22. Norouzbeigi, S.; Vahid-Dastjerdi, L.; Yekta, R.; Sohrabvandi, S.; Zendeboodi, F.; Mortazavian, A.M. Celiac therapy by administration of probiotics in food products: a review. Curr Opin Food Sci 2020, 32, 58-66, https://doi.org/10.1016/j.cofs.2020.01.005.

23. Benucci, I.; Cerreti, M.; Maresca, D.; Mauriello, G.; Esti, M. Yeast cells in double layer calcium alginatechitosan microcapsules for sparkling wine production. Food chem 2019, 300, https://doi.org/10.1016/j.foodchem.2019.125174.

24. Chaudhary, H.J.; Patel, A.R. Microencapsulation technology to enhance the viability of probiotic bacteria in fermented foods: An overview. Int J Ferment Foods 2019, 8, 63-72, https://doi.org/10.30954/2321712X.02.2019.1.

25. Moradi, M.; Mardani, K.; Tajik, H. Characterization and application of postbiotics of Lactobacillus spp. on Listeria monocytogenes in vitro and in food models. LWT- Fodd Sci Technol 2019, 111, 457-464, https://doi.org/10.1016/j.lwt.2019.05.072.

26. Pradeep Prasanna, P.H.; Charalampopoulos, D. Encapsulation in an alginate-goats' milk-inulin matrix improves survival of probiotic Bifidobacterium in simulated gastrointestinal conditions and goats' milk yoghurt. Int J Dairy Technol 2019, 72, 132-141, https://doi.org/10.1111/1471-0307.12568.

27. Afzaal, M.; Saeed, F.; Arshad, M.U.; Nadeem, M.T.; Saeed, M.; Tufail, T. The effect of encapsulation on the stability of probiotic bacteria in ice cream and simulated gastrointestinal conditions. Probiotics Antimicro 2019, 11, 1348-1354, https://doi.org/10.1007/s12602-018-9485-9.

28. Chávarri, M.; Marañón, I.; Ares, R.; Ibáñez, F. C.; Marzo, F.; del Carmen Villarán, M. Microencapsulation of a probiotic and prebiotic in alginate-chitosan capsules improves survival in simulated gastro-intestinal conditions. Int J Food Microbiol 2010, 142, 185-189, https://doi.org/10.1016/j.ijfoodmicro.2010.06.022.

29. Buts, J.P.; Keyser, N.D. Transduction pathways regulating the trophic effects of Saccharomyces boulardii in rat intestinal mucosa. Scand $J$ Gastroenterol 2010, 45, 175-185, https://doi.org/10.3109/00365520903453141.

30. Conrads, G.; Bockwoldt, J.A.; Kniebs, C.; Abdelbary, M.M. Health-Associated Niche Inhabitants as Oral Probiotics: the case of Streptococcus dentisani. Front Microbiol 2018, 9, https://doi.org/10.3389/fmicb.2018.00340. 